\title{
Erratum: Time-dependent spin and transport properties of a single-molecule magnet in a tunnel junction [Phys. Rev. B 94, 054311 (2016)]
}

\author{
H. Hammar and J. Fransson
}

(Received 7 December 2018; published 21 December 2018)

\section{DOI: 10.1103/PhysRevB.98.219907}

The authors would like to make the following changes to the article:

(1) In later works we realized that the local magnetic occupation could be of importance in the calculations. We also noted a sign error in the local field due to the charge flow. This results in Eq. (3) changing to

$$
\mathbf{B}^{\text {eff }}(t)=\mathbf{B}+\frac{v}{g \mu_{B}} \mathbf{m}(t)-\frac{1}{e g \mu_{B}} \int \boldsymbol{\epsilon} \mathbf{j}\left(t, t^{\prime}\right) d t^{\prime} .
$$

Here, the sign in front of the local field due to the charge flow was changed, and the local magnetic occupation, defined as $\mathbf{m}(t)=\langle\mathbf{s}(t)\rangle=\frac{1}{2}\left\langle\psi(t)^{\dagger} \boldsymbol{\sigma} \psi(t)\right\rangle=\frac{1}{2} \operatorname{Imsp} \boldsymbol{\sigma} \mathbf{G}^{<}(t, t)$, was added. This results in Eq. (A3) being rewritten as

$$
\begin{aligned}
\mathcal{S}= & \mathcal{S}_{\mathrm{WZWN}}-g \mu_{B} \int \mathbf{B}(t) \cdot \mathbf{S}^{q}(t) d t-v \int \mathbf{m}(t) \cdot \mathbf{S}^{q}(t) d t+\frac{1}{e} \int \boldsymbol{\epsilon} \mathbf{j}(t) \cdot \mathbf{S}^{q}(t) d t \\
& +\frac{1}{e} \int \mathbf{S}^{q}(t) \cdot \mathbb{J}\left(t, t^{\prime}\right) \cdot \mathbf{S}^{c}\left(t^{\prime}\right) d t d t^{\prime}+\frac{1}{e} \int \mathbf{S}^{q}(t) \cdot \mathbb{J}^{K}\left(t, t^{\prime}\right) \cdot \mathbf{S}^{q}\left(t^{\prime}\right) d t d t^{\prime},
\end{aligned}
$$

and Eq. (B4) as

$$
\mathbf{B}^{\mathrm{eff}}(t)=\mathbf{B}+\frac{v}{g \mu_{B}} \mathbf{m}(t)-\left[\frac{1}{e g \mu_{B}} \int \boldsymbol{\epsilon} \mathbf{j}\left(t, t^{\prime}\right) d t^{\prime}+\frac{1}{e g \mu_{B}} \int \mathbb{J}\left(t, t^{\prime}\right) d t^{\prime} \mathbf{S}(t)\right] .
$$

(2) In the correction to the quantum dot Green's functions we noted the term $\left(\mathbf{g}_{1} \cdot\langle\mathbf{S}\rangle\right) \mathbf{g}_{1}$ was missing. It means that Eq. (21b) should be

$$
\delta \mathbf{G}_{1}\left(t, t^{\prime}\right)=-v \oint_{C}\left\{g_{0}\langle\mathbf{S}\rangle g_{0}+\left(\mathbf{g}_{1} \cdot\langle\mathbf{S}\rangle\right) \mathbf{g}_{1}+i\left[\mathbf{g}_{1} \times\langle\mathbf{S}\rangle\right] g_{0}+i g_{0}\left[\langle\mathbf{S}\rangle \times \mathbf{g}_{1}\right]+i\left[\mathbf{g}_{1} \times\langle\mathbf{S}\rangle\right] \times \mathbf{g}_{1}\right\} d \tau,
$$

and Eq. (C2a) should be

$$
\begin{aligned}
\delta \mathbf{G}_{1}^{</>}\left(t, t^{\prime}\right)= & -v \int\left\{g_{0}^{r}\langle\mathbf{S}\rangle g_{0}^{</>}+g_{0}^{</>}\langle\mathbf{S}\rangle g_{0}^{a}+\left(\mathbf{g}_{1}^{r} \cdot\langle\mathbf{S}\rangle\right) \mathbf{g}_{1}^{</>}+\left(\mathbf{g}_{1}^{</>} \cdot\langle\mathbf{S}\rangle\right) \mathbf{g}_{1}^{a}+i\left[\mathbf{g}_{1}^{r} \times\langle\mathbf{S}\rangle\right] g_{0}^{</>}+i\left[\mathbf{g}_{1}^{</>} \times\langle\mathbf{S}\rangle\right] g_{0}^{a}\right. \\
& \left.+i g_{0}^{r}\left[\langle\mathbf{S}\rangle \times \mathbf{g}_{1}^{</>}\right]+i g_{0}^{</>}\left[\langle\mathbf{S}\rangle \times \mathbf{g}_{1}^{a}\right]+i\left[\mathbf{g}_{1}^{r} \times\langle\mathbf{S}\rangle\right] \times \mathbf{g}_{1}^{</>}+i\left[\mathbf{g}_{1}^{</>} \times\langle\mathbf{S}\rangle\right] \times \mathbf{g}_{1}^{a}\right\} d \tau
\end{aligned}
$$

(3) The retarded/advanced Green's functions in Eqs. (15) and (17) have the opposite signs, and they should be minus/plus in front of the equations.

(4) We found two small errors in the code. One was where $\Gamma_{\sigma}$ was used in the exponent of the time-dependent retarded/advanced Green's functions instead of $\Gamma_{\sigma} / 2$. This gave the fields and current in the time-dependent fields smaller amplitudes for a given exchange coupling $v$. The other error was a double counting of the Fermi functions in the time-dependent currents resulting in twice the amplitude.

(5) We noticed that the paper included a few typographical errors. In Eq. (5) the whole expression should be divided by 4. The charge current in Eq. (24a) should have a negative sign. At the end of Sec. III C, the expressions should read " $\Gamma_{0} /\left(1+\Gamma_{0}^{2}\right)^{4}$ and $\Gamma_{0}^{3} /\left(1+\Gamma_{0}^{2}\right)^{4}$." The right expressions were included in the calculation and thus do not change the overall results.

Most of the errors were fixed in the derivative work Ref. [1], but unfortunately two remained. First, Eqs. (9) and (11) of the paper missed the term for the local magnetic occupation $\mathbf{m}$, although, it was included in the calculations. Second, the sign error for the retarded/advanced Green's function remained in the calculations for the paper.

The errors are of minor importance and do not change the overall results of the paper. Correcting Eqs. (3) and (21b) of the paper and the errors in the numerical calculations give the same qualitative behavior, although, there are some small quantitative differences.

The authors thank C. Verdozzi for pointing out the last typographical error. 